\title{
Working memory in chess
}

\author{
T. W. ROBBINS, E. J. ANDERSON, D. R. BARKER, A. C. BRADLEY, \\ C. FEARNYHOUGH, R. HENSON, and S. R. HUDSON \\ University of Cambridge, Cambridge, England \\ and \\ A. D. BADDELEY \\ M.R.C. Applied Psychology Unit, Cambridge, England
}

\begin{abstract}
Three experiments investigated the role of working memory in various aspects of thinking in chess. Experiment 1 examined the immediate memory for briefly presented chess positions from master games in players from a wide range of abilities, following the imposition of various secondary tasks designed to block separate components of working memory. Suppression of the articulatory loop (by preventing subvocal rehearsal) had no effect on measures of recall, whereas blocking the visuospatial sketchpad (by manipulation of a keypad) and blocking the central executive (by random letter generation) had equivalent disruptive effects, in comparison with a control condition. Experiment 2 investigated the effects of similar secondary tasks on the solution (i.e., move selection) of tactical chess positions, and a similar pattern was found, except that blocking the central executive was much more disruptive than in Experiment 1. Experiment 3 compared performance on two types of primary task, one concerned with solving chess positions as in Experiment 2, and the other a sentence-rearrangement task. The secondary tasks in each case were both designed to block the central executive, but one was verbal (vocal generation of random numbers), while the other was spatial in nature (random generation of keypresses). Performance of the spatial secondary task was affected to a greater extent by the chess primary task than by the verbal primary task, whereas there were no differential effects on these secondary tasks by the verbal primary task. In none of the three experiments were there any differential effects between weak and strong players. These results are interpreted in the context of the workingmemory model and previous theories of the nature of cognition in chess.
\end{abstract}

Thinking in chess is of particular interest to the psychologist, in that it can be represented both visuospatially - as a sequence of moves on a chessboard - and propositionally - either according to various types of notation or as verbal protocols, such as those collected by early researchers (e.g., de Groot, 1965). Variation in these modes of representation could conceivably distinguish strong from weak players, and even underlie these individual differences in chess skill. Milojkovic (1982) has proposed that stronger players rely to a greater extent on propositional coding, supporting the claim with a demonstration that the time taken by a master to decide whether a particular piece was under attack is independent of the physical details of the problem, such as the spatial separation of the pieces, whereas the latencies of a group of weaker players were affected by such spatial features.

Holding (1985) has criticized the emphasis on spatial processing by pointing out the verbal components of chess and referring to evidence that top players have been reported to exhibit superior verbal skills, as manifested particularly by their competence in acquiring foreign languages and their prevalence in professional writing occupations.

We thank Pertii Saariluoma for helpful discussions. Correspondence should be addressed to T. W. Robbins, Department of Experimental Psychology, Downing Street, Cambridge CB2 3EB, U.K.
Pfau and Murphy (1988) found that a verbal knowledge test of chess skill correlated better with tournament ratings than did a test of visual memory. In addition, it was noted by Holding (1989a) that some of the classic work of Chase and Simon (1973a, 1973b) on chess memory could also be interpreted from a verbal perspective; the large differences between experts and amateurs were found following verbal presentation of positions, as well as following their visual presentation. Finally, Holding (1989b) attempted to block the possible verbal mediation of analysis of chess positions by having players count backward in threes while inspecting positions - a task that might be expected to impede subvocal articulation. He found that this additional task severely impaired the quality of the moves selected.

However, within the context of contemporary models of working memory (Baddeley, 1986), Holding's results can be interpreted in a quite different manner. If working memory is considered as a set of satellite, specialized short term memory systems, coordinated by a central executive (CE), there is considerable evidence to suggest that the task of counting backward in threes is sufficiently complex not only to suppress the so-called articulatory loop (AL), but also to impair the functioning of the CE itself.

Several studies have now shown that it is possible to dissociate different components of working memory by the use of appropriate secondary tasks. For example, verbal reasoning has been shown to be sensitive to the blockade 
of the AL by the repetition of simple words such as the (Baddeley \& Hitch, 1974), whereas visual pursuit tracking is significantly disrupted by blocking the visuospatial sketchpad (VSSP) (Baddeley, 1986; Baddeley, Grant, Wight, \& Thomson, 1975; Logie, 1986). While identifying the essential features of the CE has proven to be a more problematic task, there is considerable evidence to suggest that, operationally speaking, the generation of random strings of letters demands resources that would normally be attributed to such a mechanism (Baddeley, 1986).

Consequently, in this study, we sought to apply the working-memory model to the issue of the nature of thought processes occurring in chess players varying in strength from the level of weak club player to the level of master and grandmaster. To use chess as a model for human cognition has the advantage that there is a sophisticated unidimensional rating system for accurately measuring the level of chess skill (see Holding, 1985). In Experiment 1, we concentrated on immediate memory for chess positions. This has, of course, been the subject of seminal investigations by de Groot (1965) and Chase and Simon (1973b). However, there has been little investigation of chess memory in terms of the working-memory model outlined above. We explicitly compared the effects of blocking the $\mathrm{AL}$, the VSSP, and the CE on the memory for briefly exposed chess positions. In Experiment 2, we extended the study to include those processes responsible for move selection in positions with tactical solutions. Finally, in Experiment 3 , we compared the effects on solving tactical chess positions of tasks that block the $\mathrm{CE}$ by utilizing primarily verbal processes with tasks that utilize nonverbal processes. The specificity of these effects was assessed by comparing them with results obtained for a primary task requiring the rearrangement of words to form sentences.

\section{EXPERIMENT 1 \\ Effects of Secondary Tasks on Memory for Chess Positions}

\section{Method}

\section{Subjects}

Twenty chess players, recruited from the Cambridge City and Cambridge University Chess Clubs, were used as subjects. They were all male, and ranged in ranking from BCF (British Chess Federation) Grade 130 to BCF Grade 240. This spans chess-playing strengths from weak club player to grandmaster; experts are generally rated from 175 to 200 , and masters are rated above 200 . Grandmasters are generally rated at about 240 , and a world chess champion, such as Kasparov, at above 280 . The 20 subjects were divided into two groups - weaker players $(n=12$, gradings 130-174), and stronger players $(n=8$, gradings 181-240). Eighteen of the subjects were 30 years of age or less.

\section{Materials and Procedure}

Twenty chess positions were selected from obscure master games, some being modified slightly. All positions were taken from the middle game phases and consisted of 16 pieces. A listing of the positions is given in the Appendix. The experiment took place in a quiet room. The subject sat between two chessboards, with a partition in front of the left-side board. Full sets of white and black chess pieces were placed on either side of the right-side board. The procedure used was similar to that of de Groot (1965). One of the 20 positions was set up manually behind the partition, which was then removed to allow the subject to see the board. After a 10-sec study period, the partition was replaced, obscuring the test position from the subject, who then recalled the position by placing pieces on the right-side board. There was no formal time limit for recall, but subjects were encouraged to take not more than $1 \mathrm{~min}$. After recall was completed, the attempt at reconstruction was noted before the boards were cleared for the next position. Each of the four test conditions comprised four positions, the ordering of which was determined by a $4 \times 4$ Latin square. Thus, each position was used in each condition, across subjects. The positions were presented to the subject alternately from the vantage of the white and the black pieces. Before testing began, four practice trials were run, one for each of the four distracting conditions described below. The whole test session generally took about $70 \mathrm{~min}$.

\section{Test Conditions and Design}

(1) Control: The control condition was run exactly as described above, with no distracting secondary task. (2) Articulatory-loop suppression: The subject was required to repeat the word the in time with a metronome adjusted to a frequency of one per sec. The secondary task was begun before the partition was raised, and continued throughout the 10-sec exposure period and the time taken for recall. (3) Visuospatial-sketchpad suppression: The subject performed a secondary task that employed a calculator keyboard, comprising a matrix of $4 \times 4$ keys. He reconstructed the position with his preferred hand and with his other hand completed a self-paced, preassigned sequence of keypresses, throughout the trial. The sequence of keypresses was to press the four keys in each row from left to right before moving to the next row. When the fourth row was completed, the subject returned to the top row. The keyboard was out of sight of the subject, beneath the table, and he was not allowed to look at it during the trial. A record was kept of the sequences used from the printer attached to the calculator. (4) Central-executive suppression: The subject was asked to generate a random string of letters of the alphabet aloud, in time with the metronome set at a rate of one per sec. The sequence of letters generated was recorded for later analysis. It was emphasized to the subject that the letter string should be random, and this was explained carefully when necessary (Baddeley, 1966).

\section{Scoring}

Each board was scored using a system derived from de Groot (1965), the object being to describe recall performance in terms of a single score. Thus one point was awarded for the correct piece in the correct position; one point was deducted for any error (i.e., the wrong piece in the correct position, the correct piece in the wrong position, or the wrong piece in the wrong position). Bonus points were awarded in certain circumstances; for example, if two or more pieces bore the correct topographical relationship to one another, but were one file or row misplaced, one point was awarded for two pieces in the correct relationship but wrong position, two points were awarded for three pieces, three points for four pieces, and so on.

The other measures taken were of secondary-task performance in the VSSP and CE conditions. In the VSSP condition, the percentage of incorrect keypresses was noted. In the CE condition, the randomness of the letter strings was analyzed using a BASIC program that calculated Shannon's measure of information for single events $(H)$ and pairs of events [ $H$ (pairs); see Shannon \& Weaver, 1949]; that is, the average uncertainty associated with an event or pair of events occurring was calculated, according to the following formulae

$$
H=\sum_{i=1}^{c} p(i) \log _{2} p(i),
$$

where $c=$ number of outcomes and $p(i)=$ probability of Outcome $i$; and

$$
H(\text { pairs })=-\sum_{i j} p(i, j) \log _{2} p(i, j),
$$

where $p(i, j)=$ probability of Outcome $i$ followed by Outcome $j$. 
Analysis of variance (ANOVA) was used to examine memory performance across conditions, to compare memory performance between the two groups, and to investigate any interaction between chess strength and test condition. Subsidiary-task performance was also analyzed using ANOVA. Linear regression was also used to analyze the relationship between chess-playing strength (BCF grading) and memory performance.

\section{Results}

\section{Retention of Chess Positions}

Figure 1 shows the effects of the various conditions on recall performance. There was a significant effect of condition on memory performance $[F(3,54)=6.83, p<.01]$. Post hoc comparisons using Dunnett's $t$ test showed that the VSSP and CE blocking conditions were both significantly different from the control condition, but the $\mathrm{AL}$ suppression condition was not. As expected, there was a significant effect of strength, with players in the stronger group performing better than those in the weaker group $[F(1,18)=12.13 ; p<.001]$, but there was no significant interaction between strength and condition $[F(3,54)=1.08$, n.s.]. Mean values for stronger players were control, 40.4; AL, 37.1; VSSP, 16.3; and CE, 20.9; for weaker players, they were control, $2.9 ; \mathrm{AL}, 3.3$; VSSP, -4.8 ; and CE, -9.8 . There was a significant positive correlation between recall performance and chess-playing strength $(r=.78, p<.01)$.

\section{Secondary-Task Performance}

Visuospatial-sketchpad task. There was wide variation in performance on this task, but there were no significant differences between the two groups $[F(1,16)<1.0$, n.s.]. The weaker group scored an average of 22.7 (range $=8.3-64.1)$ incorrect keypresses, and the stronger group scored an average of 15.8 (range $=6.2-57.7)$.

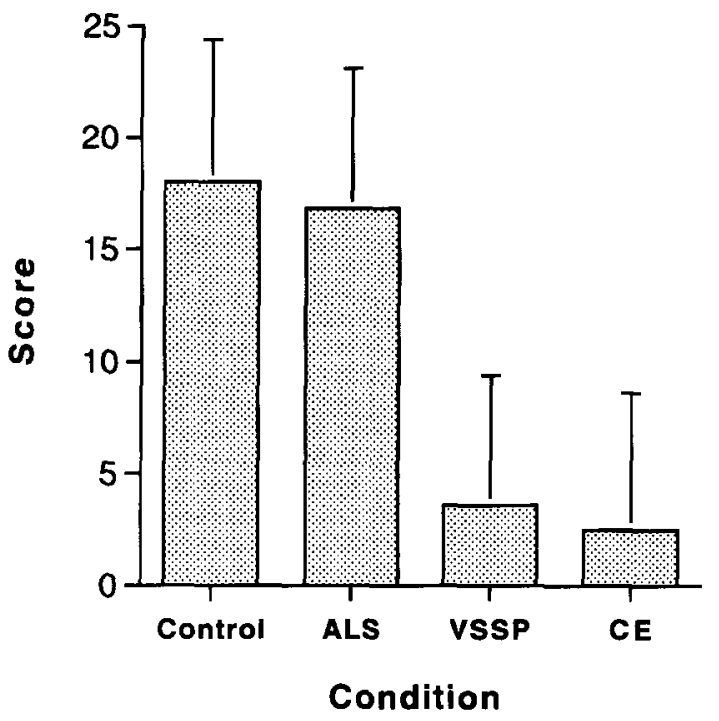

Figure 1. The effects of various secondary tasks on memory recall of chess problems. Secondary tasks: ALS, articulatory suppression; VSSP, visuospatial sketchpad [suppression]; CE, central executive [suppression]. It should be noted that the score shown on the ordinate can potentially fall in the negative range. See text for further details.
Central-executive task. There were no significant differences between the stronger and weaker groups in their capacities to generate random letters during the primary task. The weaker group had mean values of 2.7 and 3.42 for the $H$ and $H$ (pairs) variables, respectively, compared with corresponding values for the stronger group of 2.85 and 3.58. Neither of these differences was significant $[H$ : $F(1,18)=1.02 ; H$ (pairs): $F(1,18)=0.74]$.

\section{Discussion}

The results were clear-cut. Memory for chess positions was determined by chess strength and by the nature of the imposed secondary task, but there was no interaction, nor even a trend for such an interaction, between these factors. The replication of de Groot's (1965) finding was not surprising, but the fact that stronger players were superior independently of the nature of the distracting task suggests that their superior skill is not a consequence of greater or lesser utilization of the components of working memory in this task. The lack of an interaction between skill and secondary task is apparently not simply a result of adjustments in performance on the VSSP and CE secondary tasks, as the level of performance of neither of these differed between the two groups. The lack of an interaction between chess strength and performance should be tempered by the rather weak statistical power of the present design for detecting such an interaction; for a medium-sized effect, it was only about $20 \%$ (Cohen, 1969). However, Cohen has pointed out that the assessment of interactions frequently lacks power, and this possible failing is diminished by the absence of any obvious trend for the interaction to reach significance.

It was possible to degrade recall performance in two of the three secondary-task conditions - namely, VSSP and CE. This was in itself slightly unexpected because of the earlier work by Charness (1976) and Frey and Adesman (1976), which had seemed to suggest that memory for chess positions, being rather insensitive to interference by concurrent tasks, was not greatly dependent upon short term memory. However, in both cases, the lack of interference occurred when the activity (such as counting backward in threes in the latter study) was interpolated in the delay period between presentation and recall, whereas in the present study, the subjects were required to perform the interfering tasks during the presentation of the positions, with essentially no delay before recall began.

Blocking the AL by repetition of the word the did not affect performance, suggesting that memory for briefly exposed chess positions does not, after all, depend very much upon verbal processing. It cannot be argued that the task produced an insufficient blockade of the $\mathrm{AL}$, because its effects have been well documented using similar testing parameters, in other situations (Baddeley, 1986). By contrast, operating a keypad in a fixed spatial sequence produced a marked impairment of memory. This task presumably blocked the operation of the VSSP, although confounding effects of motor interference may have been produced as the subjects attempted to replace the chess pieces in the recall phase. It was, of course, impossible to 
employ some of the more conventional means of blocking the VSSP, such as by use of visuospatial tracking (Baddeley et al., 1975) or irrelevant pictures (Logie, 1986), because of their direct visual interference with the presentation of the chess positions. However, Saariluoma (1992) has recently reported that a variation of the Brooks (1968) letter task-in which subjects are asked to imagine themselves walking around the edges of a letter from a defined point and to report whether the corners they contact require turns to the left or right - produced marked impairments in two different chess tasks, one of which involved counting the minor pieces on a board. Thus, the disruptive effects generalize across different ways of blocking the operation of the VSSP. In addition, the Brooks task has been shown to disrupt other forms of visuospatial processing, which are not affected by tasks such as AL suppression (Baddeley et al., 1975). Overall, these findings converge to suggest a strong modularity in the processes that subserve many aspects of cognition in chess.

A decrement in performance comparable to that resulting from keypad operation was produced by the generation of random letters, a secondary task that can be argued to block the CE. This occurred even in the strongest players. Thus, although our grandmaster subject scored as many as 64 points in the control condition, his lowest score (55) was in the $\mathrm{CE}$ condition. A possible interpretation of the apparent involvement of the $\mathrm{CE}$ is that it is vital for the continued processing of the positions of the chess pieces that may be encoded largely in visuospatial terms. One way in which the CE could clearly influence memory for chess pieces is by providing access to structures in long term memory store, such as typical pawn formations, which may facilitate the spatial encoding of the positions. Such an involvement does not preclude the possibility that the memory traces undergo transformations to other, nonspatial, codes, also available from long term memory. In general agreement with Chase and Simon's (1973b) viewpoint, it seems plausible that the stronger players have access to more encoding strategies, resulting from their richer experience of chess. This is also consistent with the finding that strong players make much better use of semantic features of chess positions (e.g., by consideration of the best next move and the weighing up of the various strategic goals) than of structural ones (e.g., pawn formations and the occurrence of open ranks, files, and diagonals) in recognition memory for chess positions (Goldin 1978). This also avoids the assumption that chess skill depends on superior short term memory for chess positions, as Chase and Simon's (1973a, 1973b) position implies.

It also would seem likely that occupying the $\mathrm{CE}$ with an irrelevant task seriously affects the analysis of chess positions because the detailed calculation of precise lines of play requires working memory for the on-line storage of sequences of moves, as well as the evaluation of the positions resulting from each line of analysis. In Experiment 2, we directly investigated the involvement of working memory in analysis of positions in which forced, tactical lines of play were possible.

\section{EXPERIMENT 2 \\ Effects of Secondary Task on Chess-Move Selection}

\author{
Method
}

\section{Subjects}

Twelve chess players, all undergraduates from Cambridge University, were used as subjects. There were 11 males and 1 female, ranging in age from 19 to 23 , and in ranking from BCF Grade 120 to BCF Grade 206. None of the subjects used in Experiment 1 participated. The 12 subjects were divided into two groups, one consisting of weaker players ( $n=6$, gradings 120-150), and the other comprising stronger players ( $n=6$, gradings 150-206).

\section{Materials and Procedure}

Test chess positions were selected carefully from Livshitz (1988; see Appendix). A variety of different problem themes was selected. The problems were all taken from actual play, and each position had a roughly equivalent number of chess pieces $(M=16.1, S D=2.92)$. Each problem allowed a forced win (i.e., arrival at a theoretically winning position) in an unspecified number of moves. Eight problems were wins for white and eight were wins for black. The subjects were told which side was to move, and hence which side had the winning move sequence. They were asked to report whether a particular position was familiar, in which case, substitute positions were to be assigned. However, this proved unnecessary.

The problems were presented in diagrammatic form, each on a separate sheet of paper, allowing the recording of the correct move sequence after study of the positions for a 3 -min period. Sixteen problems were used for the four test conditions. Each problem was used approximately equally often in each condition. The problems were roughly balanced for difficulty over the four test conditions. Performance of the secondary tasks was maintained throughout the duration of each 3-min study period. The subjects were advised that the correct solutions to the problems typically involved checkmate or the decisive gain of material in a few moves. They were requested to write down as much as possible of their analysis of the positions immediately after each 3-min study period. Thus, they were explicitly asked, "Please write down what you think is the best solution," and also, "Please write down any other continuations that you considered (in order of merit)." There was a 10 -min break after the first 8 problems, and the test session typically lasted for $2 \mathrm{~h}$.

\section{Test Conditions}

The four secondary-task conditions were similar to those used in Experiment 1, with some differences, as described below. For each subject, the order of test condition followed a different repeating pattern, intended to avoid the fatiguing effects of having two demanding tasks immediately following one another. The pattern minimized practice effects by ensuring that a task was never immediately repeated. Practice was given for each secondary task, and baseline measurements were taken before and after the main experiment. The two baselines were averaged for the purpose of comparisons with the test condition.

Control condition. The subjects pressed the top left corner key of a $3 \times 3$ keypad (see below) at a rate of one per sec, cued by a metronome. The latency of each keypress was recorded by a computer program (see below).

Articulatory-loop suppression secondary task. The subjects were required to repeat the word see-saw once per second, in time to a metronome.

Visuospatial-sketchpad suppression secondary task. A $3 \times 3$ keypad was used. The subjects were required to press the eight peripheral keys in a repetitive, clockwise sequence beginning at the top left corner, at a rate of one press per sec, cued by a metronome. They used one finger of the preferred hand, and were instructed not to 
look at the keypad, except when they had wandered onto the wrong set of keys. The output of the keypad was connected to the user port of a $\mathrm{BBC}$ microcomputer, and a $\mathrm{BASIC}$ program was used to record the sequence and latency of each keypress.

Central-executive suppression secondary task. The same random letter generation task was used as in Experiment 1. However, pilot experiments showed that generating letters at the rate used in Experiment 1 (i.e., one per sec) completely disrupted problem-solving performance. Hence, the rate was adjusted to one per $2 \mathrm{sec}$ for Experiment 2.

\section{Scoring}

Livshitz (1988) provides an analysis of each problem, including the correct continuation, and this analysis formed the basis of the scoring system. In general, solutions are scored on the following basis: If a subject specified as his or her first choice not only the first move of the correct solution correctly, but also the most accurate subsequent sequence leading to a win, this scored maximum points. If a subject saw the correct first move but failed to recognize its full significance by incorrectly proposing an aiternative solution as the first-choice continuation, this received some credit. Failure to mention the correct first move at all resulted in no points being awarded. Points were awarded for each problem as follows: Correct first move plus accurate continuations for both white and black (as first-choice solution by the subject) $=10$; correct first move, but inaccurate continuation for either side (first choice) $=5$ (minimum), increasing to 9 , depending on the amount of correct detailed solution provided; correct first move plus accurate continuation (but not the first-choice continuation by the subject) $=3$; correct first move, but incorrect continuation (and not as first choice by the subject) $=1$; failure to mention correct first move $=0$. The maximum score obtainable was 160 points.

\section{Statistical Analysis}

The methods used were similar to those used in Experiment 1.

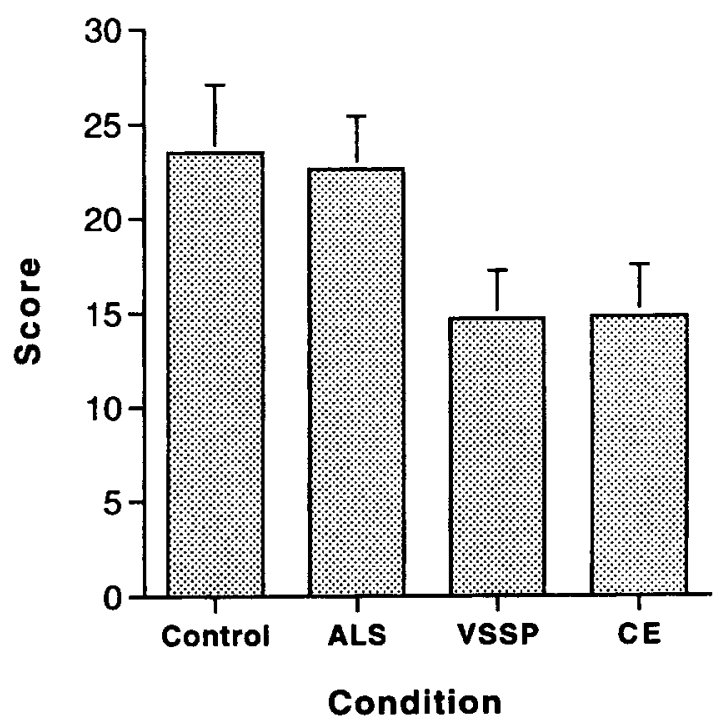

Figure 2. Effects of secondary tasks on move selection, as measured by the solution of tactical chess positions. Secondary tasks: ALS, articulatory suppression; VSSP, visuospatial sketchpad [suppression]; CE, central executive [suppression]. Mean (SEM) values are shown.

\section{Results}

\section{Solution of Tactical Chess Problems}

Figure 2 shows the mean scores under each condition. There was a significant effect of condition on problemsolving performance $[F(3,30)=3.47, p<.05]$. Post hoc application of Dunnett's test showed that both the VSSP and CE secondary-task conditions differed significantly from the control condition, with the AL secondary-task condition showing no significant difference. As expected, the stronger group differed significantly from the weaker group $[F(1,10)=15.5, p<.01]$, but there was no significant interaction between strength and condition $[F(3,30)<1.0$, n.s.]. Mean scores for the stronger and weaker groups were: stronger: control $=30.3, \mathrm{AL}=27.8, \mathrm{VSSP}=19.0$, $\mathrm{CE}=19.0$; weaker: control $=16.7, \mathrm{AL}=17.3, \mathrm{VSSP}=$ $10.3, \mathrm{CE}=10.7$. There was a significant positive correlation between chess-playing strength and tactical problemsolving performance $(r=.70, p<.01)$.

\section{Secondary-Task Performance}

Control task. The variance between keypress intervals was taken as a measure of performance accuracy. For each subject, the average baseline variance was greater than that in the test condition.

Visuospatial-sketchpad suppression secondary task. A summary of the performance measures for the VSSP task is provided in Table 1 (Rows 3 and 4). For errors, there was a significant deterioration in performance between baseline and test $[F(1,10)=16.96, p<.01]$, but no significant main effect of strength $[F(1,10)<1.0]$. A similar pattern was found for the variance measure [baseline-test deterioration, $F(1,10)=5.34, p<.05$; strength, $F(1,10)<$ 1.0]. Importantly, for both measures, the deterioration from baseline to test was equivalent in the weaker and stronger groups [strength $\times$ retest interactions, both $F \mathrm{~s}(1,10)<$ 1.0].

Central-executive suppression secondary task. The mean values for the two main indices of random letter generation, $H$ and $H$ (pairs), for the weaker and stronger groups, are shown in Table 1. For both measures, there was a significant worsening of performance from baseline to test $[F(1,10)=29.1$ and 30.1, respectively, $p<.001]$. However, there were no significant main effects of strength, and the degree of impairment from baseline to test was equivalent in both groups [both $F \mathbf{s}(1,10)<1.0$, n.s.].

\section{Discussion}

Possibly the most dramatic finding in this experiment arose from the pilot observations of a complete inability to perform the primary task of chess analysis under the conditions employed in Experiment 1 for random letter generation. Given that the slower rate of random letter generation actually used in this experiment nevertheless produced substantial impairments in tactical analysis, this strongly implies that move selection in chess positions is more dependent on the $\mathrm{CE}$ than is memory for chess positions. Pre- 
Table 1

Performance of the Secondary Tasks in Experiment 2

\begin{tabular}{|c|c|c|c|c|}
\hline \multirow[b]{3}{*}{ Condition } & \multicolumn{4}{|c|}{ Player Strength } \\
\hline & \multicolumn{2}{|c|}{ Weaker } & \multicolumn{2}{|c|}{ Stronger } \\
\hline & Baseline & Test & Baseline & Test \\
\hline \multicolumn{5}{|l|}{ Controi } \\
\hline$M$ & 79.05 & 100.93 & 34.24 & 99.42 \\
\hline$S D$ & 114.3 & 116.2 & 11.56 & 38.12 \\
\hline \multicolumn{5}{|c|}{ VSSP secondary task } \\
\hline Errors $(M)$ & 0.25 & 4.26 & 0.58 & 3.00 \\
\hline Variance $(M)$ & 72.44 & 118.16 & 76.13 & 149.9 \\
\hline \multicolumn{5}{|l|}{ CE secondary task } \\
\hline \multicolumn{5}{|l|}{$H$} \\
\hline$M$ & 3.557 & 3.413 & 3.457 & 3.318 \\
\hline$S D$ & 0.059 & 0.094 & 0.075 & 0.072 \\
\hline \multicolumn{5}{|l|}{$H$ (pairs) } \\
\hline$M$ & 4.427 & 4.369 & 4.38 & 4.317 \\
\hline$S D$ & 0.036 & 0.035 & 0.04 & 0.035 \\
\hline
\end{tabular}

sumably, it is precisely the search, evaluation, and decision processes necessary for move selection that are central to thinking in chess (cf. Holding, 1985) and that are susceptible to blockade of the CE. The fact that some players were able to solve some positions under CE blockade most likely arose from the incomplete nature of the suppression; those subjects were therefore able to time-share performance of the letter-generation task with analysis of the positions.

Mutual interference by the tasks of letter generation and chess-position solution was evident from the decrements seen in the performance of the secondary task in the test compared with its baseline periods. It is again to be noted that the degree of disruption was broadly the same for the stronger and weaker players; the CE was not engaged to a greater or lesser extent in strong players, showing that their superior performance could not be attributed to the differential use of this component of working memory.

The pattern of impairment seen for the other two secondary tasks, involving, respectively, suppression of the $\mathrm{AL}$ and suppression of the VSSP, was very similar to that seen in Experiment 1 and in the experiments of Saariluoma (1992), thus supporting the general conclusions reached previously. Indeed, these conclusions are strengthened by several other considerations. First, the lack of effect of the AL suppression was confirmed using a different articulatory response (repeating see-saw rather than the) that completely filled the 1-sec interresponse interval. Second, in the case of the VSSP secondary task, the possible confounding effects of motor interference in Experiment 1 were avoided in this experiment, which did not require manual placement of the chess pieces.

Thus, it can be concluded from the present results that analysis of chess positions, like their recall, seems to depend more on those processes associated with the VSSP than on those associated with the AL. This conclusion contradicts that of Holding (1989b), who considered AL suppression to be effective in disrupting move choice. However, as already mentioned, the task used in that experiment (counting backward in threes) almost certainly requires the resources of the $\mathrm{CE}$, and its disruptive effects would then be consistent with the effects of CE suppression we have described for this experiment. Overall, the results tend to support the mind's-eye hypothesis outlined by Chase and Simon (1973a) to explain thinking in chess. In terms of the working-memory model, the initial problem is temporarily encoded spatially in terms of such factors as the image of the chessboard and the spatial positions of the chess pieces. Searching for and generating moves involves the manipulation of these spatial images, whereas the selection of candidate moves and the evaluation of particular outcomes is the province of the CE. It is not possible at this stage to determine which of these main functions is particularly dependent on the integrity of the CE.

It should be stressed that we have uncovered no evidence that stronger chess players exhibit superior functioning of the VSSP. While there were not large numbers of subjects in the two subgroups of Experiment 2, leading to reduced statistical power for rejecting the null hypothesis $(<20 \%$ for a medium-sized effect; Cohen, 1969), there was no trend whatsoever for a significant interaction. The ability to manipulate the keypad was equivalent in the two groups representing different levels of chess skill, and while the performance of the secondary task was impaired relative to the baseline conditions, it was equally affected in the two groups. Saariluoma (1992) has reached a similar conclusion based on the use of the Brooks letter test with two primary tasks, one of which (perceiving checkmate) is most analogous to the tactical positions employed here.

It should also be noted that the present results do not rule out some contribution of verbal processing to chess analysis under certain conditions. For example, it is possible that when the VSSP is blocked, subjects continue to analyze positions using a more propositional and possibly verbal form of coding. It is also possible that some aspects of chess skill not assessed in these experiments - for example, strategic evaluation - do depend on verbal propositional processes. However, in the latter case, we have preliminary evidence to suggest that strategic evaluation is also susceptible to blockade of the VSSP (Burton, Gillespie, Robbins, \& Baddeley, unpublished results, 1990). Moreover, two experiments by Saariluoma (1992) have found no effects of AL suppression on counting of minor pieces on the chessboard and in perceiving checkmates, but substantial effects of blocking visuospatial processing. Thus it is becoming increasingly evident that many aspects of the cognitive processes engaged in chess are independent of the functioning of the AL but crucially dependent on processes hypothetically subserved by the VSSP.

There is, however, a rather more mundane explanation of the pattern of results in Experiments 1 and 2 which competes with a working-memory account. It could be suggested that the effects of the secondary tasks exert effects simply in proportion to their level of difficulty. Thus, the relative lack of effect of the AL-suppression task, and the greater effect of the CE-suppression task, may arise from the relative ease of repeating a word compared with repeating patterns of keypressing, which, in turn, is easier than generating random verbal material. Countering this view, appeal can be made to the considerable evidence 
suggesting that AL suppression exerts much greater effects on verbally dominated tasks than do certain tasks designed to block the VSSP (see above). To emphasize this point in the context of reasoning tasks, a recent experiment has shown that performance of a similar AL-suppression task, when the primary task is verbal syllogistic reasoning, can be shown to be more sensitive to disruption than a keypressing task very similar to the one used here (Gilhooly, Logie, Wetherick, \& Wynn, 1993).

A related problem is that the random letter-generation task, which is so devastating to the solution of tactical chess positions is, of course, highly verbal in nature. The lack of effect of suppression of the AL then presumably implies that this deficit is primarily due to its $\mathrm{CE}$ component, as hypothesized to explain Holding's (1989b) results. But presumably we would have to predict yet greater effects on chess memory or move selection using a task with both $\mathrm{CE}$ and VSSP components. Consequently, in Experiment 3, we compared the effects of such a task, requiring the random generation of keys in a spatial array with random number generation. In order to address the issue of task difficulty, we also examined the effects of these tasks on performance of a verbal task with some affinity to solving chess problems-namely, the rearrangement of jumbled words to make a sentence. As in the previous experiments, we studied the mutual interaction of these primary and secondary tasks by also carefully quantifying the degree of randomness (or stereotypy) of secondary-task performance.

\section{EXPERIMENT 3 \\ Effects of Verbal or Spatial Secondary Tasks on Chess-Move Selection and Sentence Rearrangement}

Method
Subjects
Fifteen chess players, all undergraduates from Cambridge Uni-
versity, were used as subjects. There were 14 males and 1 female,
ranging in age from 19 to 30 , and in ranking from BCF Grade 140
to BCF Grade 235 (mean $=180$ ). None of the subjects used in Ex-
periment 1 or 2 participated.

\section{Materials and Design}

For the primary task, each subject was required to solve either chess problems or sentence-rearrangement problems (sentence anagrams). Both primary tasks were performed under three conditions: (1) random keypressing; (2) random number generation; and (3) a control condition, making a total of six conditions. These three secondary tasks required responses to be made every $2 \mathrm{sec}$ throughout the study period, cued by a short tone produced by a computer. Each condition consisted of four trials (i.e., four study periods for different example problems of each problem type). To minimize practice effects, no task, either primary or secondary, was repeated over consecutive conditions. The temporal ordering of conditions was randomized to control against blocks of atypically easy or difficult problems repeatedly occurring.

Chess primary task. Twenty-four problems were taken from Livshitz (1988), including 12 used in Experiment 2 (see Appendix), chosen according to similar criteria as before. If the subject reported seeing a particular position before (by immediately tapping on the table), it was replaced with another. This hardly ever occurred. The subjects were presented with problems as before, except that when each problem was solved within the 2 -min period, it was replaced with a novel one, so that the subjects were continuously occupied in solving the problems. Scoring was as before, except that the scores were cumulated over positions for a particular 2-min trial. When subjects were ready to proceed to the next problem, they tapped the table. Written answers to each problem were collected at the end of the 2-min trial (with the problems in view).

Verbal primary task. The subjects were presented with jumbled words that had to be rearranged to form a sentence. Thirty-five sentences, each of which were 13 words in length, were used. They were printed on strips of paper and arranged into a booklet. A typical set of jumbled words, with the first word of the sentence in bold type, was: dense the that path The was untraceable so the fog was of course (The fog was so dense that the course of the path was untraceable). (Note that subjectively, it seems very difficult, if not impossible, to perform this task visually rather than verbally.) Trials were, again, $2 \mathrm{~min}$ long, and the subjects were given additional sentences to solve if they solved the first problem within the allotted time, using the same procedure as for the chess problems. The subjects wrote down their answers at the end of the 2 -min period, with the jumbled sentences in view. Scoring for the sentence anagrams was as follows: for each sentence, a maximum of 10 points was awarded if it was semantically and syntactically correct, contained all thirteen words, and was stylistically elegant (i.e., did not contain awkward phrases that would not normally be used, or was ambiguous). A maximum of 6 points was awarded for semantic correctness. For this purpose, the sentences were divided into three clauses and a subject was awarded a maximum of 2 points for each. A maximum of 2 points was scored for grammatical correctness. One point was given if the subject employed all 13 words. One point was awarded for stylistic elegance.

Secondary tasks. Random number generation. Numbers rather than letters were used (as in Experiment 2) because of the need to sample from a smaller set for the purposes of comparison with the random keypress-generation task. The computer-generated tone set the required rate at one response per $2 \mathrm{sec}$. (2) Random keypress generation. This task required the generation of a sequence of chords on a keypad consisting of four keys. Chords are defined by the $10 \mathrm{com}-$ binations of one-key or simultaneous two-key responses that can be used. Combinations of keypresses were measured via an interface to a BBC Master 128 computer running a program written in BASIC. Errors consisting of omitted or incorrect responses were recorded. The data were analyzed in exactly the same way as the random letters of Experiment 2 and the random numbers of this experiment.

For both of the secondary tasks, the subjects were given about $40 \mathrm{sec}$ of practice and then baselines of 65 responses for each secondary task at the beginning of each session. They were instructed to respond as randomly as possible and to resume responding as soon as they could if they missed or made an illegal response. Practice was given of each primary task in conjunction with the secondary tasks.

Control and baselines. To ensure that any effects observed were attributable to the $\mathrm{CE}$, it was necessary to be sure that the $\mathrm{AL}$ and VSSP were equivalently loaded in each CE secondary-task condition. Thus, in random key generation, the AL is not suppressed, and such suppression was therefore achieved by requiring the subject to say "one" at the time he or she made each response. Similarly, in the random number task, the VSSP was also occupied by having the subjects press one key each time they said a number. In the control condition, the subjects pressed one key and said "one" every $2 \mathrm{sec}$, paced by the computer-generated tone. A short break was allowed in the middle of the experiment, when 14 of the 15 subjects were asked to complete a short questionnaire about their subjective experience during the experiment. The whole experiment typically took about $2 \mathrm{~h}$. 


\section{Statistical Analysis}

The main hypotheses under test were whether the effects of secondary tasks would interact with the type of primary task, either for primary-task performance or for secondary-task performance. In order to test the former hypothesis, a $2 \times 3$ factor repeated measures ANOVA including the factors of primary task (chess vs. sentences) and secondary task (control, random numbers, and random keypresses) was carried out, with the key $F$ ratio being the primary $\times$ secondary task interaction term. The main effect of primary task was not meaningful, as it depended on different scales of measurement, each task (chess vs. sentences) utilizing arbitrary units. Therefore, it was also appropriate to use a nonparametric one-way ANOVA with Friedman's test applied to the secondary-task conditions separately for each of the two primary tasks (Siegel, 1956).

In the case of the secondary tasks (random keypressing and random numbers), the measure of redundancy, $H$, was utilized as before. Over the number of samples taken, $H$ is a more reliable measure than $H$ (pairs), and was thus used as the main index. Although results will only be presented for $H$, those obtained with $H$ (pairs) were essentially the same. As variances were not homogeneous over

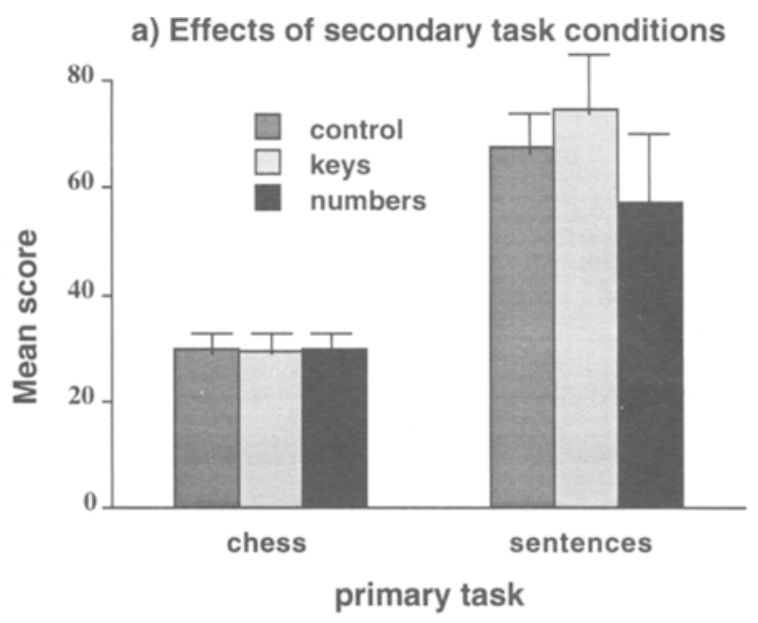

b) Effects of primary task conditions

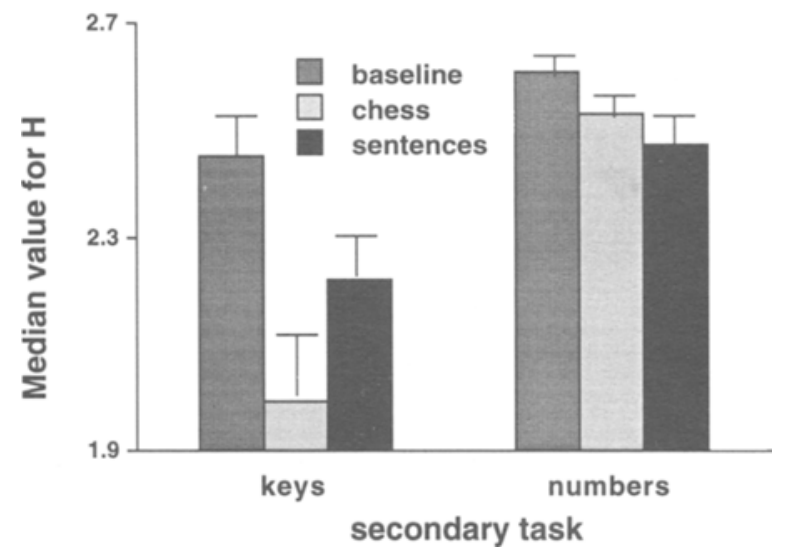

Figure 3. (a) Lack of effects of secondary tasks on primarytask performance; values shown are mean $+S E M$ values. (b) Differential effects of primary tasks on secondary-task performance. The values shown are median + interquartile-range scores. Both for the chess task and for sentence rearrangement, each of the secondary-task conditions are significantly different from one another. conditions, Friedman's nonparametric one-way ANOVA was applied to the three secondary-task conditions separately for each primary task. If an overall significant effect was detected, subsequent comparisons were based on two orthogonal contrasts using the Wilcoxon matched-pairs test (Siegel, 1956). The two contrasts were (1) baseline versus the average secondary-task score, and (2) random keys versus random numbers.

\section{Results}

\section{Effects on Primary Tasks}

For primary-task performance, there was no significant interaction between primary and secondary tasks $[F(2,28)=$ 1.3 , n.s.], following ANOVA. Thus, although from Figure $3 \mathrm{a}$ it appears that random number generation tended to impair sentence rearrangement more than random keypressing (mean scores $=57.2$ and 74.7, respectively), the concurrent random tasks had no significant differential effects on primary-task performance. This conclusion was supported by nonparametric analyses using the Friedman one-way ANOVA to compare the effects of secondary-task conditions separately for each primary task. Neither of these was significant (both $<1.0$, n.s.).

Total chess score summed over the three secondary-task conditions correlated significantly with $\mathrm{BCF}$ rating $(r=$ $.64, p<.01$ ), substantiating the significant relationship found in Experiment 2. By contrast, total-sentence score correlated negatively with $\mathrm{BCF}$ rating $(r=-.53, p<.05)$.

\section{Effects on Secondary Tasks}

Median scores for secondary-task performance are shown in Figure $3 \mathrm{~b}$. Analysis of $\mathrm{H}$ values using the Friedman test revealed a double dissociation of effects of the primary tasks on secondary-task performance. There were highly significant overall effects of both primary tasks (keys, $\chi^{2}=26.13, d f=2, p<.001$; numbers, $\chi^{2}=22.93$, $d f=2, p<.001$ ). Further analyses confirmed that performance was significantly less random than the baseline condition under secondary-task conditions involving either numbers or keys, for both primary tasks (both $T \mathrm{~s}=$ $0, n=15, p<.001$ ). More important, random keypress generation was significantly more impaired by the primary task of chess than by that of sentence rearrangement $(T=1$, $n=15, p<.001)$. In contrast, random number generation was worse when subjects were engaged in the sentencerearrangement task than when they were engaged in solving the chess positions $(T=21, n=15, p<.05)$. The latter result may seem surprising when the very small difference in median values shown in Figure $3 b$ ( 2.53 vs. 2.47 ) is considered. However, 11 out of 15 subjects showed smaller values for $H$ for random number generation with sentences than with chess, whereas only 1 out of the 15 subjects showed smaller values for random keypressing. This different pattern of effects is highly significant, according to a Fisher exact probability test $(p=.002)$. Therefore, it appears that there is strong evidence for dissociable effects of the chess-position and sentence-rearrangement tasks on secondary-task performance, with the spatial task of keypressing being more affected by the former and the verbal task of number generation being more affected by 
the latter. It was of interest to note that there was no significant correlation between performance on the random keypressing and random letter-generation tasks $(r=.16)$.

\section{Discussion}

These results complement and augment the findings of Experiment 2. The most important findings were the differential effects on secondary-task performance of the concurrent primary tasks. These have important theoretical implications, because it would appear that any account of the results obtained in Experiments 1 and 2 cannot now be framed simply in terms of a global construct such as task difficulty. The task of random keypressing was severely compromised by the requirement to find tactical solutions to chess positions, and this effect was considerably greater than the effect of a more obviously verbal task - namely, sentence rearrangement. This effect of the chess task to make random keypressing more stereotyped was also significantly greater than the effect on random number generation, a secondary task analogous to those used in Experiments 1 and 2 that had led to severe disruption of either memory for chess positions or chess-move selection. By contrast, the rearrangement of words to form a sentence reduced the randomness of number generation significantly more than did solving chess positions. This difference was much more marginal, but was nevertheless significant and in the opposite direction. The level-ofdifficulty hypothesis is also made implausible by the additional findings from the questionnaire, in which 10 out of 14 subjects reported that they found saying numbers in a random fashion to be subjectively more difficult than pressing the keys in a random sequence. Moreover, only 2 out of 14 subjects found that combining chess with random keypressing was more difficult than combining it with generating random numbers ( 4 found it to be equivalent in difficulty). These results are surprising given that random keypressing was actually more affected by the chess condition than was random letter generation. The implication is that the subjects were actually unaware of their more stereotyped performance on the keypressing task. The corollary inference is that subjective appraisal of level of difficulty of a secondary task does not necessarily correspond to its actual level of performance. The latter observation may also help to explain the lack of effects of the secondary tasks on primary-task performance, which clearly contrasts with the disruptions found in Experiments 1 and 2 . The resistance of performance to disruption by the secondary tasks suggests that the subjects were evidently paying considerable attention to the primary tasks (and so were presumably less aware of the secondary ones). While this unexpected resistance to disruption may have resulted from the differences in procedure used across the various experiments (one possibility is that generating random series of numbers is less subjectively demanding than producing random series of letters), it cannot detract from the overall conclusions. However, the variables governing the relative allocation of resources to these primary and secondary tasks should be established in further work. We were at least fortunate that the subjects appear to have behaved consistently in this respect-if not between, then at least within experimentssuggesting that any biasing effects were systematic.

\section{GENERAL DISCUSSION}

The three experiments have helped to define the nature of the thought processes used in various aspects of chess which it is hoped might have a broader significance for cognitive theories of problem solving. Verbal rehearsal appears to play a relatively small role in either memory for chess positions or the processes responsible for the analysis of chess positions leading to move selection. We argue that this cannot be because AL suppression is an inherently undemanding secondary task, since it has marked effects on serial verbal recall (Baddeley, Lewis, \& Vallar, 1984), verbal reasoning (Farmer, Berman, \& Fletcher, 1986), and a complex computer-generated control task (Logie, Baddeley, Mane, Sheptak, \& Donchin, 1988). It is also more readily disrupted than a VSSP secondary task similar to that used here during syllogistic reasoning (Gilhooly et al., 1993). In contrast, performance of a rather simple motor task requiring some visuospatial processing does interfere with both memory and move selection in chess positions.

The occupation of a hypothetical CE system that plays a pivotal role of coordination and other functions in the working-memory model also has severe disruptive effects. These appear to be greater, however, when the task has spatial rather than verbal characteristics (Experiment 3). The effects of blocking the CE cannot simply be attributed to task difficulty, as the verbal secondary task had a greater effect than the spatial one when the primary task had predominantly verbal components. On the other hand, it must be pointed out that such distinctions are not absolute; the verbal task of sentence rearrangement clearly had a limited spatial component and, similarly, chess may have some verbal propositional features, that, as yet, remain elusive.

The exact role of the CE requires further elucidation, but it appears to be more heavily implicated in move selection - those processes by which sequences of moves are generated and their outcomes weighed against one another. Unpublished results of Burton et al. (1990) have shown that there is a measurable, though small, effect on the strategic evaluation of quiescent positions. Possible effects on forward search and the initial generation of promising candidate moves will require further study.

Despite the clear involvement of two of the major components of working memory in chess cognition, there was little evidence from any of the three experiments in this study to suggest that differences in chess skill depend either qualitatively or quantitatively on differences in the operation of working memory. This conclusion agrees with that of Saariluoma (1992), who used a set of conditions complementary to those of the present study. The positive relationship between chess skill and BCF rating is particularly impressive when it is realized that $\mathrm{BCF}$ rating was 
negatively related to performance on the verbal sentencerearrangement task of Experiment 3. This underlines the specificity of the cognitive expertise of the chess players assessed in this study. The apparent lack of qualitative differences in working memory may not, of course, hold for novice players who are in the process of learning how to play. But such players would probably have been unable to cope with the complexity of the chess positions of Experiments 2 and 3 . It seems that we must search for explanations of the considerable span of competence in chess skill, as exemplified by the individuals of this study, in variations in cognitive functions other than in working memory. An obvious candidate consists of the processes that contribute to the organization of chess knowledge in long term memory. Our position is then obviously consistent with the view expressed by Chase and Simon (1973a, 1973b), that stronger chess players can process information about chess more efficiently than weaker players. This is also consistent with the more recent formulations of Chase and Ericson (1982), although some limitations in a simple application of that position have been pointed out (Saariluoma, 1989). It is possible that the capacity to select moves quickly also depends on the capacity for stored representations to elicit candidate moves, as envisioned by Chase and Simon (1973a, 1973b). However, the ability to analyze the resulting variations must depend upon other capacities, such as those of working memory.

\section{REFERENCES}

BADDELEY, A. D. (1966). The capacity for generating information by randomization. Quarterly Journal of Experimental Psychology, 18, 119-129.

BADDELEY, A. D. (1986). Working memory. Oxford: Oxford University Press.

Baddeley, A. D., Grant, S., Wight, E., \& Thomson, N. (1975). Imagery and visual working memory. In P. M. A. Rabbitt \& S. Dornic (Eds.), Attention and performance V (pp. 205-217). London: Academic Press.

BaddelEy, A. D., \& Hitch, G. J. (1974). Working memory. In G. H. Bower (Ed.), The psychology of learning and motivation (Vol. 8 , pp. 47-90). New York: Academic Press.

Baddeley, A. D., Lewis, V. J., Eldridge, M., \& Thomson, N. (1984). Attention and retrieval from long-term memory. Journal of Experimental Psychology: General, 113, 211-216.

Baddeley, A. D., Lewis V. J., \& Vallar, G. (1984). Exploring the articulatory loop. Quarterly Journal of Experimental Psychology, 36, 233-252.

BRooks, L. (1968). Spatial and verbal components in the act of recall. Canadian Journal of Psychology, 22, 349-368.
Burton, J. B., Gillespie, G. M., Robbins, T. W., \& Baddeley, A. D. (1990). Executive processes and visuospatial imagery in chess. Unpublished manuscript.

Charness, N. (1976). Memory for chess positions: Resistance to interference. Journal of Experimental Psychology: Human Learning \& Memory, 2, 641-653.

Chase, W. G., \& Erikson, K. A. (1982). Skill and working memory. In G. H. Bower (Ed.), The psychology of learning and motivation (Vol. 16, pp. 1-58). New York: Academic Press.

Chase, W. G., \& Simon, H. A. (1973a). The mind's eye in chess. In W. G. Chase (Ed.), Visual information processing (pp. 215-281). New York: Academic Press.

Chase, W. G., \& Simon, H. A. (1973b). Perception in chess. Cognitive Psychology, 4, 55-81.

COHEN, J. (1969). Statistical power analysis for the behavioural sciences. New York: Academic Press.

DE Groot, A. D. (1965). Thought and choice in chess. The Hague: Mouton:

EuWE, M., \& Kramer, H. (1965). The middle game in chess (Vol. 2). London: Bell.

Farmer, E. W., Berman, J. V. F., \& Fletcher, Y. L. (1986). Evidence for a visuo-spatial scratch-pad in working memory. Quarterly Journal of Experimental Psychology, 38A, 675-688.

FreY, P. W., \& ADESMAN, P. (1976). Recall memory for visually presented chess positions. Memory \& Cognition, 4, 541-547.

Gilhooly, K. J., Logie, R. H., WETherick, N. E., \& WinN, V. (1993). Working memory and strategies in syllogistic-reasoning tasks. Memory \& Cognition, 21, 115-124.

Goldin, S. (1978). Memory effects for the ordinary: Typicality effects in chess memory. Journal of Experimental Psychology: Human Learning \& Memory, 4, 605-611.

Holding, D. H. (1985). The psychology of chess skill. Hillsdale, NJ: Erlbaum.

Holding, D. H. (1989a). Adversary problem solving by humans. In K. J. Gilhooly (Ed.), Human and machine problem solving (pp. 83-122). New York: Plenum.

Holding, D. H. (1989b). Counting backward during chess move choice. Bulletin of the Psychonomic Society, 27, 421-424.

Livshitz, A. (1988). Test your chess $I Q$ (Book 1). Oxford: Pergamon.

LOGIE, R. H. (1986). Visuospatial processing in working memory. Quarterly Journal of Experimental Psychology, 38A, 229-247.

Logie, R. H., Baddeley, A. D., MANe, A., Sheptak, R., \& Donchin, E. (1988). Working memory in the acquisition of complex skills. In A. M. Colley \& J. R. Beech (Eds.), Cognition and action in skilled behaviour (pp. 361-377). Amsterdam: Elsevier

MiLojkovic, I. D. (1982). Chess imagery in novice and master. Journal of Mental Imagery, 6, 125-144

PfaU, H. D., \& MURPhy, M. D. (1988). Role of verbal knowledge in chess. American Journal of Psychology, 101, 73-86.

SAARILUOMA, P. (1989). Chess players' recall of auditorily presented chess positions. European Journal of Cognitive Psychology, 1, 309-320.

SAARILUOMA, P. (1992). Visuospatial and articulatory interference in chess players' information intake. Applied Cognitive Psychology, 4, 309-320.

SHANnon, C. E., \& WeAver, W. (1949). The mathematical theory of communication. Urbana, IL: University of Illinois Press.

SIEGEL, S. (1956). Non-parametric statistics. New York: McGraw-Hill.

\section{APPENDIX}

Experiment 1: Stimulus Material. Positions 1-16 in Conventional Algebriac Notation

1. White: Kf1,Rb6,a5,d6,e3,f2,g3,h3 Black: Ke6,Rc5,a6,c4,e4,f5,g6,h5

2. White: Kd3,Be3,a2,b2,c4,f2,g3,h2 Black: Kc6,Bd8,a , a5,e6,f7,g7,h7

3. White: $\mathrm{Kg} 1, \mathrm{Ne} 2, \mathrm{a} 3, \mathrm{~b} 3, \mathrm{e} 4, \mathrm{f2}, \mathrm{g} 2$,h2 Black: $\mathrm{Kc} 7, \mathrm{Be} 5, \mathrm{a} 7, \mathrm{~b} 7, \mathrm{eb}, \mathrm{f} 7, \mathrm{f6}, \mathrm{h} 7$

4. White: $\mathrm{Kg} 1, \mathrm{Qd} 3, \mathrm{a} 5, \mathrm{~b} 4, \mathrm{c3}$, f2,g3,h2 Black: $\mathrm{Kg} 8, \mathrm{Qe} 6, \mathrm{a} 6, \mathrm{~b} 7, \mathrm{c6}, \mathrm{f} 7, \mathrm{~g} 4, \mathrm{~h} 5$

5. White: Kf2,Nf3,a2,c2,c3,d3,f4,g3 Black: Ke8,Ba4,a7,b6,c5,d5,f6,g7

6. White: $\mathrm{Kg} 1, \mathrm{Rf} 1, \mathrm{a} 3, \mathrm{~b} 2, \mathrm{~d} 4, \mathrm{f4}, \mathrm{g} 5, \mathrm{~g} 2$ Black: $\mathrm{Kg} 7, \mathrm{Rh} 8, \mathrm{a} 7, \mathrm{~b} 7, \mathrm{~d} 5, \mathrm{e} 6, \mathrm{f} 7, \mathrm{f5}$

7. White: Kf1,Rd1,Rel,a5,b2,c2,f2,g2,h2 Black: Kh8,Ra8,Nc5,Bd6,a6,b5,h7

8. White: $\mathrm{Kh} 1, \mathrm{Be} 3, \mathrm{Bg} 2, \mathrm{Nf} 3, \mathrm{a3}, \mathrm{b4}, \mathrm{c} 4, \mathrm{~d} 3$, f2,h2 Black: Kg8,Re8,Rd8,b6,c5,d6

9. White: $\mathrm{Kh} 2, \mathrm{Rc} 8, \mathrm{Bb} 3, \mathrm{Bc} 5, \mathrm{c4}, \mathrm{g} 3, \mathrm{~h} 3$ Black: $\mathrm{Kg} 7, \mathrm{Ra} 3, \mathrm{Bf} 6, \mathrm{Nf} 2, \mathrm{e} 4, \mathrm{f} 7, \mathrm{~g} 6, \mathrm{~h} 7, \mathrm{cc}$ 
10. White: Kf 2,Qf5,Be3,e4,f3,g3,h2 Black: Kh7,Qe7,Nd8,b5,c6,c7,f7,g7,h6

11. White $\mathrm{Kg} 1$,Qd2,Rf1,Rf7,e6,b2,c2,g2 Black: Kg7,Qh4,Ra8,Bd5,Nd8,Ng8,g4,h5

12. White: Ke3,Rc3,Nb3,a3,b4,e4,f4,g3 Black: Kd6,Rb8,Nd7,a6,b7,e5,f6,g5

13. White: Kd2,Rc3,Be4,Ne5,b4,f2,g3,h2 Black: Ke7,Rb8,Nd8,Be8,a6,b7,e6,f7

14. White: Kg2,Qe6,Rd4,Nd6,a2,b3,f2,g2 Black: Kh7,Qe7,Ra8,Rf8,a7,b6,g7,h5

15. White: Kc1,Qc3,Re2,Nd1,Bg5,a3,b2,c2 Black: Ka8,Qd7,Rc8,Bd6,Nf6,a6,b7

16. White: Kf2,Qa8,Bd6,a2,b3,e2,f3,g3 Black: Kg7,Qf 7,Nf8,a6,b5,f6,g5,h5

17. White: $\mathrm{Kg} 1$,Qe2,Rd1,Nb6,Nc4,a2,b3,f2,g2 Black: Kg8,Qb5,Re8,Bf6,Ng5,h5,g7

18. White: $\mathrm{Kg} 1, \operatorname{Re} 1, \mathrm{Ba} 1, \mathrm{Bg} 2, \mathrm{Nd} 4, \mathrm{~d} 5, \mathrm{f} 2, \mathrm{~g} 3$ Black: Kg8,Rc8,Ba6,Ba3,Na2,b5,g7,h7

19. White: Kg2,Rd1,Re1,Bc3,Bc2,b3,f3,g4 Black: Ke7,Rc8,Rd8,Ne6,Nf6,b6,d6,f7

20. White: Kf4,Nb4,a2,b2,e2,f3,g4,h4 Black: Ke6,Nc5,a7,b6,d4,f6,g6,h7

Experiment 2: Stimulus Material. Positions 1-16

1. White*: Ke2,Qh5,Rb7,Bf4,a3,e6,f2,g3,h3 Black: Kd8,Qh1,Rc8,Ra8,Bf8,a6,c7,d5,e7,g5,h6

2. White: Kf1,Qe2,Re1,Bf2,Bf3,a3,b4,c7,d5,e4 Black*: Kg8,Qh2,Rf8,Bc8,Ng4,a5,b6,e5,h6

3. White*: Kg1,Qd7,Rb5,Bb2,Nf7,a2,f2,g3,h2 Black: Kh7,Qa4,Ra8,Rg8,Bg7,Nb3,a7,b7,g6,h6

4. White: Kg2,Qb2,Ra7,a6,c5,e3,f2,g3,h2 Black*: Kh7,Qh5,Rf3,c6,d5,e4,g7,h4

5. White*: Ke5,Rb1,Rc8,c6,g5 Black: Kf7,Rc3,Bd5,e6,e4,f5,h7

6. White: Kh2,Qd6,Re3,Be5,b3,c4,g2,h3 Black*: Kh8,Qa2,Rg8,Bg7,a7,b6,c5,h7

7. White*: Kh2,Qd6,Ra7,Ba3,b5,e4,g2,h3 Black: Kg8,Qg6,Re8,Bc2,f6,g7,h7

8. White: Kh1,Qc4,Ra1,Rfl,Nh5,a2,c3,d6,f3,g2,h2 Black*: Kf8,Qb6,Rb2,Rg6,Nd3,a6,d7,f7,f6,h7

9. White*: Kh2,Qf 3,Rf 7,a3,b2,c3,g5 Black: Kg8,Qb8,Re6,a6,e7,g6

10. White: Kg6,Qe3,Bf3,Ne2,d5,g5 Black*: Kg8,Qh3,Rh4,g7

11. White*: Kb1,Qe6,Ral,Be3,b2,b3,c2,g5 Black: Kc8,Qd7,Rd5,Be7,a6,b7,g7

12. White: Kh1,Rc5,Rd2,a2,c2,c4,g2,h3 Black*: Ke6,Rg8,Be4,a6,b5,f5,f3,h4

13. White*: Kf1,Qg5,Rd1,Rd2,Be5,a2,b2,f6,h2 Black: Kh8,Qb7,Rg8,Rf 7,Bc5,a7,c4,e4,h7

14. White: Kg1,Qd3,Bg5,a5,b4,d4,f2,h4 Black*: Kh7,Qf 7,Nc4,a6,b5,d2,g6

15. White*: Ke3,Bg2,Ne4,a3,b5,c5,f4,h2 Black: Ke6,Bg3,Nd8,a7,b7,e5,f7, h6

16. White: $\mathrm{Kc2}, \mathrm{Rb} 2, \mathrm{Nd} 3, \mathrm{a} 2, \mathrm{e} 2, \mathrm{f} 2, \mathrm{~g} 3, \mathrm{~g} 4$ Black*: Kg7,Ra4,Nd5,a6,e6,f7,h3

Experiment 3 used the positions of Experiment 2 plus additional examples; contact the first author for details.

*Side to move. 